\title{
A teoria triangular do amor de Sternberg e o modelo dos cinco grandes fatores
}

\author{
Bruna Gomes Mônego - Universidade Federal do Rio Grande do Sul, Porto Alegre, Brasil \\ Maycoln Leoni Martins Teodoro' - Universidade Federal de Minas Gerais, Belo Horizonte, Brasil
}

\begin{abstract}
Resumo
O presente estudo investigou a influência dos traços de personalidade de acordo com o modelo dos Cinco Grandes Fatores (CGF) e do amor segundo a Teoria Triangular do Amor de Sternberg na satisfação conjugal. A amostra foi composta por 192 universitários que vivenciavam um relacionamento amoroso classificado como Ficante, Namoro/Noivado ou Casamento. Os participantes responderam coletivamente a Escala Fatorial de Satisfação em Relacionamento de Casal, a Escala Triangular do Amor de Sternberg e a Bateria Fatorial de Personalidade. Os resultados indicaram padrões diferentes de correlação para cada tipo de relacionamento e o modelo de Regressão Linear Múltipla foi significativo $(F=23,19, g l=10, p<0,001)$ e explicou $58 \%$ da variância. Os construtos intimidade, paixão e realização contribuíram positivamente para a satisfação, enquanto que o traço neuroticismo apresentou influência negativa. O componente compromisso e os demais traços de personalidade não obtiveram significância estatística.

Palavras-chave: Satisfação Conjugal, Modelo dos Cinco Grandes Fatores, Teoria Triangular do Amor de Sternberg.
\end{abstract}

\section{Sternberg's triangular theory of love and the big five factor model}

\begin{abstract}
This present study investigated the influence of personality traits according to the Big Five Factors (CGF) model and to love according to Sternberg's Triangular Theory of Love in marital satisfaction. Sample was composed by 192 undergraduate students who experienced a loving relationship classified as an informal relationship, dating/engagement or wedding. Participants answered the "Escala Fatorial de Satisfação em Relacionamento de Casal", the Sternberg's Triangular Love Scale and the "Bateria Fatorial de Personalidade". Data showed different patterns of correlation for each type of relationship and multiple linear regression model was significant $(F=23.19, d f=10, p<0.001)$ and explained $58 \%$ of the variance. The intimacy, passion and conscientiousness constructs contributed positively to the satisfaction, while neuroticism had a negative influence. The commitment component and the other personality traits did not reach statistical significance.

Keywords: Marital Satisfaction, Big Five Factor Model, Sternberg's Triangular Theory of Love.
\end{abstract}

O relacionamento conjugal vem recebendo grande atenção da psicologia nas últimas décadas. Os estudos publicados englobam desde teorias e modelos sobre a satisfação conjugal e teorias do amor (Falcke, Diehl, \& Wagner, 2002; Lee, 1976; Rubin, 1970), assim como investigações empíricas que associam o amor a variáveis como história familiar (Donnellan, LarsenRife, \& Conger, 2005) e bem-estar subjetivo (Kim \& Hatfield, 2004). O presente estudo pretende contribuir para a compreensão da satisfação conjugal em casais a partir da percepção relacionamento amoroso e de características de personalidade. Para tanto, será realizada uma breve apresentação sobre a Teoria Triangular do Amor de Sternberg (1986), a satisfação conjugal e o modelo dos Cinco Grandes Fatores (CGF) da personalidade.

A Teoria Triangular do Amor (Sternberg, 1986) caracteriza esse sentimento a partir de três componentes, intimidade, paixão e decisão/compromisso, que formam os vértices de um triângulo. Sternberg define intimidade como a presença

${ }^{1}$ Endereço para correspondência:

Universidade Federal de Minas Gerais - FAFICH

Departamento de Psicologia

Av. Antonio Carlos, 6627, Pampulha, Belo Horizonte, MG, Brasil, CEP 31270-901.

E-mail:mlmteodoro@hotmail.com de felicidade, respeito, entendimento mútuo, capacidade de entregar-se, apoio emocional, comunicação e valorização. Interações baseadas isoladamente nesse componente caracterizam relações semelhantes à amizade. A paixão diz respeito à atração física e sexual, à vontade de estar junto e ao romance, indicando uma união com grande excitação. Decisão/compromisso, por sua vez, está relacionado à decisão de amar e à vontade de que a relação se mantenha em longo prazo. Quando isolado, revela um relacionamento que tende a durar, mas principalmente pela influência de fatores externos, pois a paixão e a intimidade não estão presentes. Esse tipo de união também é chamado de amor vazio (Sternberg, 1986, 1997).

A presença dos três componentes estabelece o amor pleno, enquanto que a junção de dois deles indica outras formas de amar. A combinação entre intimidade e paixão, chamado de amor romântico, significa que mesmo próximos e desejando estar junto, o casal não tem a certeza de que isso será possível. Intimidade e decisão/compromisso (companheirismo) fazem com que os parceiros permaneçam unidos mesmo após o término do desejo sexual. Por fim, a paixão com decisão/compromisso (amor factual) é como o amor à primeira vista no qual existe a atração física e a vontade 
de permanecer juntos, mas o casal ainda não desenvolveu intimidade (Sternberg, 1986, 1997).

Alguns estudos sugerem que aspectos do amor contribuem para a satisfação com o relacionamento. Karwowski-Marques (2008), por exemplo, sugeriu que a percepção de intimidade de um dos cônjuges influencia a satisfação do outro. Complementariamente, Hernandez e Oliveira (2003) indicaram que aspectos relacionados à intimidade, comunicação, confiança e excitação física foram os preditores mais fortes de satisfação conjugal. Tais estudos corroboram com Sternberg (1989) uma vez que o autor estabelece que a intimidade é o maior preditor de satisfação no relacionamento.

A satisfação conjugal, por sua vez, é definida por alguns autores como sendo a avaliação que o próprio sujeito faz sobre o seu relacionamento e sobre os ganhos que ele tem com esse (Wachelke, Andrade, Cruz, Faggiani, \& Natividade, 2004). Essa avaliação depende, dentre outros aspectos, da história romântica do indivíduo, de modo que suas experiências passadas são modelos de comparação e influenciam suas expectativas sobre o relacionamento presente (Bystronski, 1995). Dessa forma, a satisfação possui variações durante a história dos relacionamentos, não se apresentando, portanto, de maneira estagnada (Olson, 2000).

A literatura tem citado diversos preditores de satisfação conjugal. Alguns dizem respeito a características sociodemográficas como escolaridade, número de filhos, nível socioeconômico e cultural e crença religiosa. Outros estão associados às experiências românticas anteriores ou ainda a construtos como proximidade, intimidade, coesão, estabilidade conjugal e estilos de amor. Há também aspectos cognitivos como estratégias adequadas de resolução de problemas e habilidade de comunicação (Norgren, Souza, Kaslow, Hammerschmidt, \& Sharlin, 2004; Olson, 2000; Sharlin, Kaslow, \& Hammerschmidt, 2000; White, Hendrick, \& Hendrick, 2004). Complementarmente, tem-se enfatizado o papel de aspectos individuais como a personalidade na satisfação conjugal (Engel, Olson, \& Patrick, 2002; Malouff, Thorsteinsson, Schutte, Bhullar, \& Rooke, 2010; Wan, Luk, \& Lai, 2000).

Quanto à personalidade, uma das teorias que mais tem sido empregada em sua avaliação e compreensão é o modelo dos CGF baseado nas teorias fatoriais e de traços (Digman, 1990; Nunes, 2005; Silva, Schlottfeldt, Rozenberg, Teles-Santos, \& Lelé, 2007). O CGF apresenta cinco dimensões que constituem a personalidade, traduzidas como extroversão (extraversion), socialização (agreeableness), realização (conscientiousness), neuroticismo (neuroticism) e abertura à experiência (openness to experience) (McCrae \& John, 1992, Nunes, Hutz, \& Nunes, 2010). Cada dimensão engloba características que vão de um pólo a outro. Sendo assim, o fator extroversão descreve desde indivíduos falantes, otimistas e sociáveis até os reservados, independentes e sóbrios. A socialização se refere tanto a pessoas afáveis, empáticas e interessadas em ajudar os demais, quanto àquelas inescrupulosas, desconfiadas e egoístas. Realização está associada a traços como responsabilidade, disciplina, zelo, honestidade e tenacidade, assim como à falta de ambição e/ou objetivos, hedonismo e, muitas vezes, distração. $\mathrm{O}$ neuroticismo aponta para os aspectos emocionais do indivíduo, principalmente quanto à forma como ele percebe e se comporta diante do sofrimento emocional. Altos escores nesse fator indicam vulnerabilidade, sintomas depressivos e ansiosos e estratégias de coping desadaptativas. Por fim, pessoas com alto índice de abertura à experiência são mais curiosas, criativas e versáteis. Já no outro pólo, elas podem preferir rotinas, ser rígidas, austeras, convencionais e apegadas a hábitos ou a valores tradicionais (McCrae \& John, 1992; Nunes, 2000, 2005; Silva et al., 2007; Vasconcelos \& Hutz, 2008).

Resultados de uma pesquisa de Fisher e McNulty (2008) indicaram impacto negativo do traço neuroticismo na satisfação conjugal e sexual de casais. De maneira semelhante, White et al. (2004) relataram que o mesmo traço foi o principal preditor negativo de satisfação e de intimidade conjugal. $O$ fator realização, por outro lado, foi apontado como preditor de todos os componentes do amor da Teoria Triangular do Amor de Sternberg (1986) e da satisfação para homens no estudo de Engel et al. (2002). No grupo feminino, a mesma dimensão foi preditora de intimidade, paixão e, juntamente com extroversão, de satisfação. Os autores sugerem que os indivíduos com escores altos no traço realização sejam mais engajados, empenhados e motivados em seus relacionamentos.

Embora tenha crescido o número de pesquisas nas últimas décadas sobre os relacionamentos amorosos, os resultados ainda são muito distintos. Percebe-se que a personalidade e o amor contribuem para a satisfação conjugal, mas poucos estudos incluem esses três construtos em suas análises (Engel et al., 2002; White et al., 2004).

Desse modo, o presente trabalho se propõe a investigar algumas características sociodemográficas da amostra (tipo de relacionamento, sexo e local onde reside) e associações entre a satisfação conjugal, os componentes do amor da Teoria Triangular de Sternberg (1986) e os traços de personalidade de acordo com o modelo CGF. Por fim, também serão analisados preditores da satisfação conjugal. Assim, o 
presente trabalho tem como hipóteses: (a) o neuroticismo será preditor negativo de satisfação conjugal e (b) os componentes do amor serão preditores positivos de satisfação conjugal.

\section{Método}

\section{Participantes}

Participaram desse estudo 198 universitários de diferentes áreas do conhecimento (psicologia, engenharia, jornalismo, ciências sociais, relações públicas, economia, serviço social e administração) que estavam vivenciando alguma relação amorosa. Destes, seis foram excluídos da amostra por não terem classificado seus relacionamentos em Ficantes, Namoro/Noivado ou Casamento. Assim, totalizou 192 participantes dos quais $72(37,3 \%)$ viviam no estado de Alagoas e 121 (62,7\%) no Rio Grande do Sul. A amostra foi separada de acordo com o tipo de relacionamento a fim de investigar possíveis diferenças. A Tabela 1 descreve o sexo, a idade e o tempo de relacionamento dos participantes.

Tabela 1 - Descrição dos participantes $(n=192)$

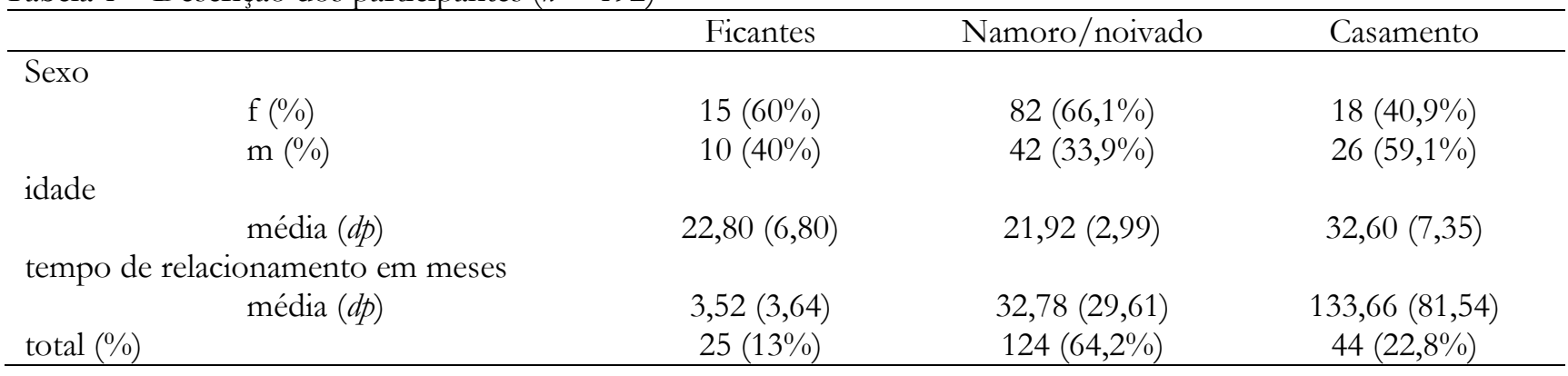

\section{Instrumentos}

Escala Triangular do Amor de Sternberg (ETAS, Sternberg's Triangular Love Scale).

Avalia a percepção do indivíduo sobre o seu relacionamento amoroso de acordo com a Teoria Triangular do Amor (Sternberg, 1986). A escala é composta por 45 itens divididos em três subescalas referentes aos construtos intimidade, paixão e decisão/compromisso. Para cada item, o participante responde o quanto cada afirmativa é verdadeira em uma escala do tipo Likert que varia de um (de jeito nenhum) a nove (extremamente). No Brasil, alguns estudos (Cassepp-Borges \& Teodoro, 2007, 2009; Gouveia, Fonseca, Cavalcanti, Diniz, \& Dória, 2009, Sternberg, 1997) investigaram as propriedades psicométricas da ETAS e de sua versão abreviada e indicaram estrutura fatorial adequada e índices satisfatórios de consistência interna. Os Alphas de Cronbach obtidos na presente pesquisa foram 0,93, 0,96 e 0,91 para os componentes intimidade, decisão/compromisso e paixão, respectivamente.

Escala Fatorial de Satisfação em Relacionamento de Casal (EFS-RC).

A EFS-RC foi desenvolvida no Brasil com o intuito de avaliar a satisfação conjugal (Wachelke et al., 2004). A escala possui nove itens que são respondidos em uma escala do tipo Likert que varia de um (discordo fortemente) a cinco (concordo fortemente) pontos. Possui dois fatores chamados Satisfação com Atração Física e Sexualidade (SAFS) e Satisfação com Afinidades de Interesses e Comportamentos (SAIC).
Em um estudo complementar, Wachelke, Andrade, Souza e Cruz (2007) encontraram Alpha de Cronbach para a escala total de 0,90 . No presente estudo, esse valor foi de 0,69 .

Bateria Fatorial da Personalidade (BFP).

Avalia a personalidade de acordo com o modelo CGF. Possui 126 itens que são pontuados em uma escala do tipo Likert de sete pontos, na qual um representa "Absolutamente não me identifico com a frase" e cinco significa "Descreve-me perfeitamente". Os dados psicométricos relacionados à estrutura fatorial e à consistência interna foram adequados, apresentando Alphas de Cronbach de 0,89, 0,84, 0,85, 0,83 e 0,74 para neuroticismo, extroversão, socialização, realização e abertura à experiência, respectivamente (Nunes, Hutz, \& Nunes, 2009). Na presente investigação os índices foram de $0,90,0,85,0,79,0,80,0,69$, respectivamente.

\section{Procedimentos}

Primeiramente foi feito contato com as universidades e, em seguida, com os coordenadores de cursos de graduação que conduziam aos professores. A aplicação foi realizada coletivamente em sala de aula e durou cerca de 45 minutos.

Esse estudo foi aprovado pelo Comitê de Ética em Pesquisa. Todos os participantes que concordaram em participar dessa pesquisa assinaram o Termo de Consentimento Livre e Esclarecido.

Análise de Dados 
Para análise de dados, foram realizadas correlações de Spearman e Teste $t$ e Análise de Variância One-Way (ANOVA) para comparação entre os grupos. Para investigação de preditores da satisfação conjugal foi utilizada Análise de Regressão Linear Múltipla com método Enter. Para afirmar que existe relação entre duas variáveis, foram consideradas as correlações moderadas a fortes. O nível de significância adotado foi de $p<0,05$.

\section{Resultados}

\section{Diferenças entre os grupos}

Análises comparativas para sexo e estado dos participantes foram realizadas com o Teste $t$. A idade média $(t(190)=-3,97 ; p<0,001)$ e tempo médio de relacionamento $(t(133,99)=-2,10 ; p<0,05)$ dos homens foram superiores aos das mulheres. O grupo feminino, por sua vez, apresentou média superior ao masculino no traço socialização $(t(185)=2,52 ; p<$ $0,05)$. Em relação ao local de coleta, houve diferenças quanto à idade $(t(191)=3,72 ; p<0,001)$ e quanto ao tempo de relacionamento $(t(179,19)=3,77 ; p<0,001)$, sendo que as médias dos participantes do Rio Grande do Sul foram maiores do que as de Alagoas. Dentre os fatores de personalidade, a amostra de Alagoas apresentou média superior a do Rio Grande do Sul no traço neuroticismo $(t(186)=-2,66 ; p<0,01)$ e inferior nos traços socialização $(t(102,84)=2,24 ; p<0,05)$ e realização $(t(108,19)=3,53 ; p<0,01)$.

Além disso, realizou-se Análise de Variância One-Way (ANOVA) para comparar os tipos de relacionamentos quanto a idade, tempo de relacionamento, traços de personalidade e componentes do amor. A Tabela 2 mostra as diferenças significativas encontradas.

Tabela 2 - Análise de Variância One-Way para o tipo de relacionamento

\begin{tabular}{|c|c|c|c|c|}
\hline & ficantes & $\begin{array}{c}\text { namoro/ } \\
\text { noivado }\end{array}$ & casamento & $\begin{array}{c}\text { anova } \\
(p<0,001)\end{array}$ \\
\hline \multicolumn{5}{|l|}{ idade } \\
\hline média $(d p)$ & $22,80(6,80)$ & $21,92(2,99)$ & $32,60(7,35)$ & $f(2)=78,97$ \\
\hline \multicolumn{5}{|l|}{ tempo de relacionamento } \\
\hline média $(d p)$ & $3,52(3,64)$ & $32,78(29,61)$ & $133,66(81,54)$ & $f(2)=95,95$ \\
\hline \multicolumn{5}{|l|}{ satisfação conjugal } \\
\hline média $(d p)$ & $41,67(4,43)$ & $45,77(4,07)$ & $44,41(4,72)$ & $f(2)=9,70$ \\
\hline \multicolumn{5}{|l|}{ socialização } \\
\hline média $(d p)$ & $133,88(24,16)$ & $144,57(18,97)$ & $148,85(16,81)$ & $f(2)=4,67$ \\
\hline média $(d p)$ & $97,50(22,27)$ & $104,55(13,88)$ & $112,05(12,75)$ & $f(2)=7,58$ \\
\hline \multicolumn{5}{|l|}{ intimidade } \\
\hline média $(d p)$ & $106,92(22,04)$ & $133,46(14,79)$ & $129,93(17,11)$ & $F(2)=27,24$ \\
\hline \multicolumn{5}{|l|}{ Decisão/compromisso } \\
\hline média $(d p)$ & $73,00(29,86)$ & $124,72(20,12)$ & $128,43(14,85)$ & $F(2)=70,95$ \\
\hline média $(d p)$ & $61,80(17,83)$ & $80,86(12,92)$ & $77,50(11,74)$ & $F(2)=20,99$ \\
\hline
\end{tabular}

O teste post hoc de Bonferroni demonstrou que os participantes casados eram mais velhos do que os demais e possuíam escores mais altos no traço realização. O grupo Ficantes, por outro lado, apresentou médias inferiores aos outros grupos quanto ao tempo de relacionamento, satisfação conjugal, socialização, intimidade, decisão/compromisso e paixão.

\section{Satisfação, Amor, Personalidade e Tipo de Relacionamento}

Padrões de correlações entre traços de satisfação conjugal, amor e personalidade foram diferentes pra cada tipo de relacionamento amoroso. $\mathrm{Na}$ Tabela 3 estão descritos os escores de Spearman para os grupos Ficantes, Namoro/Noivado e Casamento.

Como pode ser visto na Tabela 3, houve correlações moderadas e fortes em todos os grupos, embora a grande maioria seja moderada. Pode-se perceber que a satisfação conjugal, assim como seus fatores, apresentaram associações moderadas com todos os componentes do amor nos grupos Namoro/Noivado e Casamento. 
Tabela 3 - Correlações de Spearman entre satisfação, componentes do amor e traços de personalidade

\begin{tabular}{|c|c|c|c|c|c|c|}
\hline & Paixão & Intimidade & decisão & satisfação & Saic & safs \\
\hline \multicolumn{7}{|l|}{ Ficante } \\
\hline extroversão & $0,43^{*}$ & 0,29 & 0,22 & 0,36 & 0,17 & 0,28 \\
\hline socialização & 0,22 & 0,21 & 0,09 & 0,31 & 0,12 & 0,28 \\
\hline realização & 0,27 & $0,47 *$ & 0,21 & 0,29 & $0,52^{* *}$ & $-0,06$ \\
\hline neuroticismo & 0,03 & $-0,04$ & 0,18 & $-0,18$ & $-0,20$ & $-0,02$ \\
\hline abertura & $-0,25$ & 0,07 & $-0,24$ & 0,05 & 0,39 & $-0,15$ \\
\hline satisfação & $0,56 * *$ & $0,69 * * *$ & 0,35 & - & $0,69 * * *$ & $0,77 * * *$ \\
\hline saic & 0,37 & $0,78^{* * *}$ & $0,41 *$ & - & - & - \\
\hline safs & $0,42 *$ & 0,28 & 0,07 & - & 0,83 & - \\
\hline \multicolumn{7}{|l|}{ namoro/noivado } \\
\hline extroversão & 0,16 & $0,22 * *$ & 0,07 & 0,14 & 0,12 & 0,15 \\
\hline socialização & $0,24 * *$ & $0,29 * *$ & $0,26 * *$ & $0,20 *$ & $0,26 * *$ & 0,12 \\
\hline realização & 0,15 & 0,16 & 0,15 & $0,28^{* *}$ & $0,42^{* * *}$ & 0,07 \\
\hline neuroticismo & $-0,09$ & $-0,17$ & $-0,04$ & $-0,21 *$ & $-0,22^{*}$ & $-0,18^{*}$ \\
\hline abertura & 0,11 & 0,03 & $-0,10$ & $-0,01$ & $-0,01$ & 0,01 \\
\hline satisfação & $0,67 * * *$ & $0,66^{* * *}$ & $0,60 * * *$ & - & $0,84 * * *$ & $0,75^{* * *}$ \\
\hline saic & $0,53 * * *$ & $0,60 * * *$ & $0,51 * * *$ & - & - & - \\
\hline safs & $0,52^{* * *}$ & $0,44 * * *$ & $0,42 * * *$ & - & $0,29 * * *$ & - \\
\hline \multicolumn{7}{|l|}{ casamento } \\
\hline extroversão & 0,05 & 0,01 & 0,06 & 0,08 & 0,05 & 0,04 \\
\hline socialização & 0,31 & 0,56 *** & $0,47 * *$ & 0,17 & 0,02 & 0,14 \\
\hline realização & 0,11 & 0,15 & 0,02 & 0,19 & 0,06 & 0,24 \\
\hline neuroticismo & $-0,06$ & $-0,31$ & $-0,09$ & $-0,32 *$ & $-0,43^{*}$ & $-0,19$ \\
\hline abertura & 0,17 & 0,13 & 0,05 & 0,11 & 0,10 & $-0,01$ \\
\hline satisfação & $0,62^{* * *}$ & $0,72 * * *$ & $0,62 * * *$ & - & $0,82^{* * *}$ & $0,83^{* * *}$ \\
\hline Saic & $0,34^{*}$ & $0,61 * * *$ & $0,44^{* *}$ & - & - & - \\
\hline Safs & $0,69 * * *$ & $0,67 * * *$ & $0,53 * * *$ & - & $0,40 *$ & - \\
\hline
\end{tabular}

Amor e Personalidade como Preditores da Satisfação Conjugal

A investigação dos preditores de satisfação conjugal foi feita por meio de Regressão Linear Múltipla com método Enter. As variáveis independentes utilizadas para o modelo foram os componentes do amor, as dimensões da personalidade, o tipo de relacionamento, sexo e local dos participantes. Para investigar o efeito específico de cada tipo de relacionamento, as variáveis Ficantes, Namoro/Noivado e Casamento foram transformadas em medidas binárias. Os resultados estão descritos na Tabela 4.

O modelo de regressão foi adequado $(F(12)=$ 19,21; $p<0,001)$ e explicou $58 \%$ da variância da satisfação conjugal (vide Tabela 4). Dentre as variáveis independentes utilizadas, foram significativas os componentes intimidade $(\beta=0,40)$ e paixão $(\beta=0,40)$ e os traços neuroticismo $(\beta=-0,15)$ e realização $(\beta=$ $0,16)$. As demais variáveis não contribuíram significativamente para o modelo. 
Tabela 4 - Regressão linear múltipla para satisfação conjugal $(n=192)$

\begin{tabular}{lcc} 
& coeficientes padronizados & $t /$ sig \\
\cline { 2 - 3 } (constante) & beta & $6,630^{* * *}$ \\
intimidade & - & $4,38^{* * *}$ \\
compromisso & 0,40 & $-0,08$ \\
paixão & $-0,01$ & $3,84^{* * *}$ \\
neuroticismo & 0,40 & $-2,66^{* *}$ \\
extroversão & $-0,15$ & $-0,46$ \\
socialização & $-0,03$ & $-1,54$ \\
realização & $-0,10$ & $2,50^{*}$ \\
abertura & 0,16 & $-0,81$ \\
namoro/noivado & $-0,05$ & - \\
ficantes & - & $-0,63$ \\
casamento & $-0,04$ & 1,88 \\
sexo & 0,11 & $-0,40$ \\
local & $-0,02$ & 0,52 \\
\hline
\end{tabular}

Nota. ${ }^{*} \mathrm{p}<0.05,{ }^{* *} \mathrm{p}<0,01 \mathrm{e}{ }^{* * *} \mathrm{p}<0,001 ;$ Para as variáveis Namoro/Noivado, Ficante e Casamentos: $1=$ não e $2=\operatorname{sim}$.

\section{Discussão e Considerações Finais}

A presente pesquisa buscou investigar as contribuições do amor e das características individuais como a personalidade para a satisfação conjugal. A partir de análises comparativas, correlacionais e causais, foi possível verificar algumas semelhanças entre os resultados obtidos nesse estudo e os mencionados em investigações anteriores.

Não houve diferença significativa das medidas de amor e satisfação entre os grupos masculino e feminino do presente estudo. Isso também foi reportado por Engel et al. (2002) e KarwowskiMarques (2008), indicando semelhança na percepção $\mathrm{da}$ intensidade do relacionamento entre homens e mulheres. Por outro lado, algumas diferenças foram encontradas com relação ao local de coleta. No entanto, devido ao número reduzido de participantes, essas diferenças não podem ser abordadas com base nos aspectos culturais. Salienta-se também que a amostra desse estudo foi selecionada por conveniência, impossibilitando, assim, generalizações para a população.

De modo geral, os resultados apresentados indicam haver associações entre o modo como as pessoas percebem o seu relacionamento romântico e o quanto elas sentem-se satisfeitas com essa união. Alguns traços de personalidade também parecem ter uma importante participação nesse aspecto. Contudo, tais associações demonstram padrões específicos para cada tipo de relacionamento, sendo que os grupos Namoro/Noivado e Casamento apresentaram resultados mais semelhantes entre si.

As análises e discussão dos resultados das correlações têm foco nos escores com magnitude moderada a forte a fim de buscar resultados mais robustos (Dancey \& Reidy, 2006). Quanto à percepção do amor, observam-se importantes associações entre todos os seus componentes e a satisfação como já reportado na literatura (Hernandez e Oliveira, 2003; Karwowski-Marques, 2008). Essas associações são mais proeminentes nos grupos Namoro/Noivado e Casamento.

Quanto aos resultados referentes aos traços de personalidade, as análises correlacionais apontaram associações entre o traço Socialização e os aspectos românticos sugerindo que pessoas bondosas e empáticas têm percepções mais positivas sobre seus relacionamentos nos grupos casados e namoro/noivado. Isto, porém, não ocorreu no grupo Ficantes. No entanto, pode-se observar nesse grupo que pessoas mais empenhadas, confiáveis e motivadas (maior realização) relataram mais intimidade na sua relação amorosa, assim como pessoas comunicativas e assertivas (maior extroversão) percebiam mais paixão. As diferenças de resultados entre os grupos devem-se, provavelmente, ao tempo de relacionamento, o que acaba produzindo relações mais consolidadas para namorados e casados. De modo geral, os resultados desse estudo mostram que determinadas características individuais estão relacionadas com a forma de perceber a relação amorosa, sendo que essa associação é especifica para o tipo de relacionamento vivenciado.

Os dados referentes à análise de regressão destacam a paixão e a intimidade como principais preditores positivos de satisfação. Outros autores encontraram dados em comum aos relatados no presente estudo tanto para aspectos referentes à intimidade (Hernandez \& Oliveira, 2003; KarwowskiMarques, 2008; Martinez \& Fernandez, 1993) quanto 
para àqueles que dizem respeito ao envolvimento sexual (Hendrick, Hendrich, \& Adler, 1988; Hernandez \& Oliveira, 2003).

Nessa mesma direção, Norgren et al. (2004) relatam que, entre outros aspectos, a comunicação, a proximidade e a coesão apresentaram significância quando regredidos para a satisfação conjugal. Tais pontos também são incluídos no componente intimidade que, segundo Sternberg (1986), é responsável pela longa duração dos relacionamentos, assim como decisão/compromisso. No entanto, devese ter cautela quanto a algumas comparações, visto que o estudo de Norgren et al. foi realizado com casais unidos há mais de vinte anos, enquanto que a amostra da presente pesquisa é mais nova, possui relacionamentos mais curtos e inclui indivíduos que não são casados. Desse modo, é possível dizer que existem evidências de que tanto as dimensões afetivas (intimidade) quanto as de relacionamento físico (paixão) são fatores que contribuem para a satisfação conjugal.

Os dados analisados também apontaram para a influência dos fatores neuroticismo e realização na satisfação conjugal. Estudos prévios também relataram o papel negativo do neuroticismo na satisfação justificando a hipótese do presente estudo (Donnellan, Conger, \& Bryant, 2004; Fisher \& McNulty, 2008; White et al., 2004). Sugere-se que essa influência devase aos problemas relacionados à saúde mental do indivíduo, os quais são avaliados por essa dimensão. Quando em altos níveis, esse traço indica características que afetam o bem-estar subjetivo de modo geral (Nunes, Hutz, \& Giacomoni, 2009), construto correlato da satisfação conjugal.

Indivíduos com altos escores na dimensão neuroticismo tendem a apresentar ansiedade exagerada, dificuldades para tolerar frustração, impulsividade $e$ maior vulnerabilidade ao sofrimento emocional (Nunes, 2000). Além disso, evidências indicam que essas pessoas têm uma visão mais negativa das situações e tendem a se envolver em mais problemas, apresentando assim maior ocorrência de eventos estressores ao longo da vida (Watson \& Hubbard, 1996). Desse modo, suas relações interpessoais, assim como seus relacionamentos amorosos, são prejudicadas.

Quanto ao fator Realização, nota-se que ele também foi preditor de satisfação no estudo de Engel et al. (2002). Visto que para chegar a um relacionamento longo e saudável, é necessário o empenho e investimento de ambos os cônjuges em prol de um objetivo comum que seria a construção desta união (Norgren \& Cols., 2004), a falta desses esforços, como discutidos por Engel et al., podem prejudicar a relação. De forma complementar, há outras características abarcadas pela realização que podem influenciar positivamente o relacionamento conjugal como motivação e capacidade de planejamento que, por sua vez, contribui para a habilidade de resolução de problemas (Bruck \& Allen, 2003). Essa última habilidade também já foi relatada por casais satisfeitos (Norgren et al., 2004). Ademais, uma meta-análise realizada por Malouff et al (2010) sobre o modelo dos CGF e satisfação com o relacionamento também apóia os resultados obtidos no presente estudo. Dentre os cinco fatores de personalidade, apenas abertura a experiência não demonstrou associações com a satisfação com o relacionamento. As correlações foram negativas com o traço neuroticismo e positivas com realização, socialização e extroversão foram positivas.

O presente estudo contribui para a produção de conhecimento em relação à participação dos traços de personalidade e da percepção do amor na satisfação conjugal, entretanto, são necessárias pesquisas futuras para um melhor esclarecimento sobre essa temática, especialmente envolvendo ambos os cônjuges. Estudos com casais poderiam esclarecer melhor sobre os papéis das características individuais e das relacionais. Nesse sentido, se buscaria investigar de que forma as características individuais (personalidade) de cada cônjuge contribui para um relacionamento a dois.

Além disso, essa investigação fornece suporte para o desenvolvimento de estratégias terapêuticas mais eficazes no que tange ao relacionamento amoroso. Nesse sentido, salienta-se a importância de estimular as habilidades de comunicação, assim como trabalhar com a motivação, com a construção de metas comuns, com a saúde mental e com a atração física e sexual dos casais.

\section{Referências}

Bruck, C. S., \& Allen, T. D. (2003). The relationship between big five personality traits, negative affectivity, type a behavior, and work-family conflict. Journal of Vocational Behavior, 63, 457-472.

Bystronski, B. (1995). Teorias e processos psicossociais da intimidade interpessoal. Em A. Rodrigues (Org.). Psicologia social para principiantes: Estudo da interação humana. Petrópolis: Vozes.

Cassepp-Borges, V., \& Teodoro, M. L. M. (2007). Propriedades psicométricas da versão brasileira da Escala Triangular do Amor de Sternberg. Psicologia: Reflexão e Crítica, 20, 513-522.

Cassepp-Borges, V., \& Teodoro, M. L. M. (2009). Versión reducida de la escala triangular del amor: 
Características del sentimiento en Brasil. Interamerican Journal of Psychology, 43, 30-38.

Dancey, C. P., \& Reidy, J. (2006). Estatística sem matemática para psicologia - Usando SPSS para Windows. Porto Alegre: Artmed.

Digman, J. M. (1990). Personality structure: Emergence of the Five-Factor Model. Annual Review of Psychology, 41, 417-440.

Donnellan, M. B., Conger, R. D., \& Bryant, C. M. (2004). The big five and enduring marriages. Journal or Research in Personality, 38, 481-504.

Donnellan, M. B., Larsen-Rife, D., \& Conger, R. D. (2005). Personality, family history, and competence in early adult romantic relationships. Journal of Personality and Social Psychology, 88(3), 562-576.

Engel, G., Olson, K. R., \& Patrick, C. (2002). The personality of love: Fundamental motives and traits related to components of love. Personality and Individual Differences, 32, 839-853.

Falcke, D., Diehl, J. A., \& Wagner, A. (2002). Satisfação conjugal na atualidade. In A. Wagner (Ed.), A família em cena: Tramas, dramas e transformacões (pp. 172-188). Petrópolis: Vozes.

Fisher, T. D., \& McNulty, J. N. (2008). Neuroticism and marital satisfaction: The mediating role played by the sexual relationship. Journal of Family Psychology, 22(1), 112-122.

Gouveia, V. V., Fonseca, P. N., Cavalcanti, J. P. N., Diniz, P. K. C., \& Dória, L. C. (2009). Versão abreviada da Escala Triangular do Amor: Evidências de validade fatorial e consistência interna. Estudos de Psicologia (Natal), 14, 31-39.

Hendrick, S., Hendrick, C., \& Adler, N. L. (1988). Romantic relationships: Love, satisfaction, and staying together. Journal of Personality and Social Psychology, 54, 980-988.

Hernandez, J. A. E., \& Oliveira, I. M. B. (2003). Os componentes do amor e a satisfação. Psicologia Ciência e Profissão, 21(3), 58-69.

Karwowski-Marques, A. P. M. (2008). Percepcões sobre o amor, a qualidade $e$ a satisfação com o relacionamento em casais. Dissertação de mestrado não publicada, Universidade do Vale do Rio dos Sinos, São Leopoldo, RS, Brasil.

Kim, J., \& Hatfield, E. (2004). Love types and subjective well-being: A cross-cultural study. Social Behavior and Personality, 32 (2), 173-182.

Lee, J. A. (1976). The colors of love. Englewood Cliffs, NJ: Prentice-Hall.

Malouff, J. M., Thorsteinsson, E. B.,Schutte, N. S., Bhullar, N.,\& Rooke, S. E. (2010). The Five-Factor Model of personality and relationship satisfaction of intimate partners: A meta-analysis. Journal of Research in Personality, 44, 124-127.
Martinez, G. S., \& Fernandez, M. C. (1993). La teoría de Sternberg sobre el amor: Análisis empírico. Psicothema, 5(supl. 1), 151-167.

McCrae, R. R., \& John, O. P. (1992). An introduction to the five-factor model and its applications. Journal of Personality, 60, 175-216.

Norgren, M. B. P., Souza, R. M., Kaslow, F., Hammerschmidt, H., \& Sharlin, S. A. (2004). Satisfação conjugal em casamentos de longa duração: Uma construção possível? Estudos de Psicologia, 9, 575-584.

Nunes, C. H. S. S. (2000). A construção de um instrumento de medida para o fator neuroticismo / estabilidade emocional dentro do modelo de personalidade dos cinco grandes fatores. Dissertação de mestrado não publicada, Universidade Federal do Rio Grande do Sul, Porto Alegre, RS, Brasil.

Nunes, C. H. S. S. (2005). Construção, normatização e validação das escalas de socialização e extroversão no modelo dos cinco grandes fatores. Tese de doutorado não publicada, Universidade Federal do Rio Grande do Sul, Porto Alegre, RS, Brasil.

Nunes, C. H. S. S., Hutz, C. S., \& Giacomoni, C. H. (2009). Associação entre bem estar subjetivo e personalidade no modelo dos cinco grandes fatores. Avaliação Psicológica, 8(1), 99-108.

Nunes, C. H. S. S., Hutz, C. S., \& Nunes, M. F. O. (2010). Bateria Fatorial de Personalidade (BFP) - Manual técnico. São Paulo, SP: Casa do Psicólogo.

Olson, D. H. (2000). Circumplex model of marital and family systems. Journal of Family Therapy, 22, 144-167.

Rubin, Z. (1970). Measurement of romantic love. Journal of Personality and Social Psychology, 16, 265273.

Sharlin, S., Kaslow, F., \& Hammerschmidt, H. (2000). Together through thick and thin: A multinational picture of long-term marriages. Nova York: The Haworth Clinical Practice Press.

Silva, R. S., Schlottfeldt, C. G., Rozenberg, M. P., Teles-Santos, M., \& Lelé, A. J. (2007). Replicabilidade do modelo dos cinco grandes fatores em medidas da personalidade. Mosaico: Estudos em Psicologia, 1(1), 37-49.

Sternberg, R. J. (1986). A triangular theory of love. Psychological Review, 93, 119-135.

Sternberg, R. J. (1989). El triangulo del amor: Intimidad, passión y compromisso. Barcelona: Paidós.

Sternberg, R. J. (1997). Construct validation of a Triangular Love Scale. European Journal of Psychology, 27, 313-335.

Vasconcelos, S. J. L., \& Hutz, C. S. (2008). Construção e validação de uma escala de abertura à experiência. Avaliação Psicológica,7(2), 135-141. 
Wachelke, J. F. R., Andrade, A. L., Cruz, R. M. Faggiani, R. B., \& Natividade, J. C. (2004). Medida da satisfação em relacionamento de casal. Psico-USF, 9(1), 11-18.

Wachelke, J. F. R., Andrade, A. L., Souza, A. M., \& Cruz, R. M. (2007). Estudo complementar da validade fatorial da escala fatorial de satisfação em relacionamento e predição de satisfação global com a relação. Psico-USF, 12(2), 221-225.

Wan, W. W. N., Luk, C. L., \& Lai, J. C. L. (2000). Personality correlates of loving styles among Chinese students in Hong Kong. Personality and Individual Differences, 29, 169-175.
Watson, D., \& Hubbard, B. (1996). Adaptational style and dispositional structure: Coping in the context of the five-factor. Journal of Personality, 64, 737-774.

White, J. K., Hendrick, S. S., \& Hendrick, C. (2004). Big five personality variables and relationship constructs. Personality and Individual Differences, 37, 1519-1530.

Sobre os autores:

Recebido em novembro de 2010

Reformulado em janeiro de 2011

Aprovado em março de 2011

Bruna Gomes Mônego é Psicóloga graduada pela Universidade do Vale do Rio dos Sinos (UNISINOS), mestranda em Psicologia e especializanda em Neuropsicologia na Universidade Federal do Rio Grande do Sul (UFRGS). Trabalha, principalmente, com avaliação psicológica, terapia cognitivo-comportamental e construção e validação de instrumentos psicológicos.

Maycoln Leoni Martins Teodoro é Psicólogo graduado e mestre pela Universidade Federal de Minas Gerais (UFMG), doutor pela Albert-Ludwigs-Universität Freiburg (Alemanha) com estágio pós-doutoral na UFRGS. Atualmente é Professor Adjunto na Graduação e Pós-Graduação em Psicologia da UFMG. Trabalha, principalmente, com relações familiares, problemas emocionais e de comportamento, vulnerabilidade cognitiva para a depressão, pesquisa em terapia cognitivo-comportamental e construção e validação de instrumentos psicológicos. 